\title{
EL PAPEL DE LAS TECNOLOGÍAS EN LA FORMACIÓN DE LOS CUIDADORES INFORMALES DE PERSONAS MAYORES
}

\author{
Luis López-Lago Ortiz \\ https://orcid.org/0000-0002-2129-3442 \\ Universidad de Extremadura. \\ Personal Científico investigador Escuela Politécnica. \\ luislopezlago@unex.es \\ Borja Rivero Jiménez \\ https://orcid.org/0000-0002-3691-0968 \\ Universidad de Extremadura. \\ Personal Científico investigador Escuela Politécnica. \\ brivero@unex.es \\ Lorenzo Mariano Juárez \\ https://orcid.org/0000-0001-8483-7200 \\ Universidad de Extremadura. \\ Profesor Facultad de Enfermería y Terapia Ocupacional. \\ lorenmariano@unex.es \\ David Conde Caballero \\ https://orcid.org/0000-0002-5967-4371 \\ Universidad de Extremadura. \\ Profesor Facultad de Enfermería y Terapia Ocupacional. \\ dcondecab@unex.es \\ Beatriz Muñoz González \\ https://orcid.org/0000-0001-9373-5062 \\ Universidad de Extremadura. \\ Profesora Facultad de Formación del Profesorado. \\ bmunoz@unex.es
}

Para citar este artículo puede utilizar el siguiente formato:

Luis López-Lago Ortiz, Borja Rivero Jiménez, Lorenzo Mariano Juárez, David Conde Caballero y Beatriz Muñoz González: "El papel de las tecnologías en la formación de los cuidadores informales de personas mayores", Revista Atlante: Cuadernos de Educación y Desarrollo, ISSN: 1989-4155 (vol 13, № 6 julio-septiembre 2021, pp. 80-89). En línea:

https://doi.org/10.51896/atlante/ZOZO9175

\section{RESUMEN}

En el artículo se analizan las relaciones sociales y los patrones culturales que sustentan la cultura informal de los cuidados en España, y se exponen algunos de los factores principales que suponen un obstáculo para profesionalización de este sector. Se establecen los perfiles de los cuidadores informales destacando que en su mayoría son mujeres, ya sean familiares o, si no pertenecen al núcleo familiar, cabe destacar el papel de las mujeres inmigrantes en el sector no profesionalizado. Así mismo analizamos las distintas relaciones que tienen los colectivos vinculados a los cuidados informales de los mayores con las tecnologías, destacando el uso del teléfono móvil y de las aplicaciones de mensajería instantánea. Con este resultado realizamos una propuesta para que los responsables de las políticas de cuidados de las personas mayores contemplen acciones de 
formación que se puedan transmitir a través del teléfono móvil y mediante aplicaciones de mensajería con las que los potenciales usuarios estén familiarizados.

Palabras clave: Cuidadores, Envejecimiento, Tecnología, Cuidados informales, Mensajería instantánea.

\section{THE ROLE OF TECHNOLOGIES IN THE TRAINING OF INFORMAL CAREGIVERS OF THE ELDERLY}

\section{ABSTRACT}

The article analyzes the social relations and cultural patterns that sustain the informal caregiving culture in Spain and exposes some of the main factors that are an obstacle to the professionalization of this sector. The profiles of informal caregivers are established, highlighting that most of them are women, whether they are family members or, if they do not belong to the family nucleus, the role of immigrant women in the non-professionalized sector is noteworthy. We also analyzed the different relationships that the groups linked to the informal care of the elderly have with technology, highlighting cell phones and instant messaging applications. With this result, we proposed for those responsible for care policies for the elderly to consider training actions that can be transmitted through the cell phone and messaging applications with which potential users are familiar.

Keywords: Caregivers, Aging, Technology, Informal care, Instant messaging.

\section{INTRODUCCIÓN}

España, como el resto de los países occidentales, ha experimentado una serie de cambios demográficos caracterizados por el incremento de la longevidad y la disminución de la fecundidad lo que ha provocado un notable envejecimiento de la población (Abellan et al., 2017). Este fenómeno se ha visto acompañado por otros procesos sociales en las últimas décadas como son la incorporación de la mujer al mercado laboral, la migración del entorno rural a las ciudades, el descenso en los niveles de convivencia intergeneracional en el hogar, el descenso de la tasa de viudez, cambios culturales en las formas de ocio, transformaciones del modelo de familia o el fenómeno migratorio (Martinez , 2003) que afectan directamente a los procesos socioculturales relacionados con el envejecimiento. En este sentido cabe destacar que, a pesar de esos cambios, en España hay una sólida base cultural que propicia que los cuidados de los mayores se sigan dando en el ámbito familiar, en lo que se ha llamado "la cultura informal de los cuidados" y que analizaremos a continuación. Además, hay un fenómeno, que en los años 90 del siglo pasado cobró una magnitud destacable en el ámbito de los cuidados informales, la incorporación de cuidadores no profesionales de origen inmigrante (Zabalegui et al., 2007). 
EI IMSERSO (Instituto de Mayores y Servicios Sociales) hace una estimación de los cuidadores ${ }^{1}$ informales dentro del núcleo familiar que alcanzaría las 950.528 personas, y la población a la que atiende este colectivo sería de 1.226.181 personas mayores de 65 años. Así mismo, esta institución reconoce la dificultad a la hora de hacer una estimación de las personas trabajadoras informales que no pertenecen al núcleo familiar, hecho que se complica aún más en el caso de personas de origen extranjero en situación irregular (Instituto de Mayores y Servicios Sociales, 2005).

En este escenario de relaciones de cuidados informales, ¿de qué manera se puede mejorar la cualificación de los cuidadores para que su labor sea lo más profesional posible?, ¿se pueden desarrollar políticas públicas que contemplen la formación de este colectivo al que es tan difícil de acceder desde las administraciones públicas? y ¿puede la tecnología ayudar a transmitir contenidos formativos a los cuidadores informales? En este artículo realizamos un primer acercamiento a estos temas.

\section{CUIDADOS INFORMALES Y DIFICULTADES PARA LA PROFESIONALIZACIÓN EN ESPAÑA.}

La persistencia de la informalidad y las dificultades para la profesionalización del sector de los cuidados hacia las personas mayores en España tienen su origen en múltiples factores. Pero el peso cultural del modelo de cuidados donde la familia es el principal proveedor de éstos tiene un peso fundamental (Moreno-Colom et al., 2017). Existe un acuerdo generalizado en calificar este modelo de cuidados familiares como "familista" o "familiarista" (Casanova et al., 2017; Fernández-Alonso \& Ortega, 2018; García-Faroldi, 2015).

El modelo "familista" de cuidados a las personas mayores en los Estados del Bienestar, está particularmente arraigado en las sociedades del Sur de Europa o mediterráneas (Moreno, 2004). Y aunque es innegable que los cambios socioculturales experimentados por estas sociedades en las últimas décadas han transformado las formas y la intensidad de las relaciones dentro del ámbito familiar, en el modelo "familista", la familia nuclear es el principal espacio social suministrador de cuidados en términos culturales (Fernández-Alonso \& Ortega, 2018). Así este modelo se proyecta en el imaginario social como un "deber ser" de las conductas de cuidados, donde las familias tienen una suerte de "obligación moral" respecto a los cuidados de las personas mayores. En este patrón cultural el peso de los cuidados recae principalmente en las mujeres (Di Novi et al., 2015). En definitiva, la norma cultural impone un modelo ideal de cuidados donde la mujer es la responsable de proveer la atención a las personas mayores, y esto dificulta la profesionalización del sector de los cuidados en cuanto las mujeres, especialmente en entornos con un fuerte peso de la tradición, asumen de forma natural su rol de cuidadoras dentro del ámbito familiar (Recio et al., 2015).

No obstante, hay que puntualizar que hablamos de un modelo cultural "familista" que se ha visto sometido a numerosos cambios socioculturales y que está en permanente transformación. Si bien

\footnotetext{
${ }^{1}$ López (2016) define a los cuidadores informales como aquellos que no tienen un beneficio económico a cambio de desarrollar la labor cuidadora, o lo hacen dentro de la economía sumergida, por lo general no tienen un horario fijo estipulado y no tienen por qué tener una formación especializada.
} 
sus ideas principales subyacen en el sustrato ideológico de las relaciones familiares y condicionan las conductas respecto a los cuidados, y consecuentemente sobre la profesionalización de las personas cuidadoras. En este sentido cabe señalar que fenómenos como la incorporación de la mujer al mundo laboral, los cambios en las pautas de convivencia dentro de la familia nuclear o la emigración del campo a la ciudad han relativizado el peso de los "ideales de cuidados" propios del modelo "familista".

Así en España actualmente, más allá de los imaginarios culturales sobre el cuidado, encontramos que coexisten los recursos de carácter público para el cuidado de los mayores, con una multitud de formatos (residencias de mayores, teleasistencia, asistencia a domicilio, etc.), con una amplia gama de servicio que proveen empresas y personal autónomo y, en menor medida, entidades sin ánimo de lucro, como ONG, asociaciones, fundaciones, etc. (De Souza, 2019; Durán, 2015; Osorio et al., 2018). Así, en términos culturales, los cuidados de las personas mayores recaen en la familia, pero cuando ésta no puede proporcionarlos, o decide no asumirlos, son los recursos públicos quienes ocupan ese lugar. Por otra parte, donde no llega el Estado, o si por decisión propia o de la familia, no se eligen los recursos de la administración pública, hay un amplio abanico de oferta privada en la provisión de cuidados (López-Lago et al., 2021b).

Respecto a los recursos para los cuidados de personas de edad avanzada con dificultades de movilidad, enfermedades crónicas o mayores de 80 años, en España, se ha vuelto preferencial la asistencia en residencias de mayores (Abellan et al., 2017; Osorio et al., 2018). Si embargo los recursos residenciales plantean algunas problemáticas en su acceso, tales como los tiempos en la asignación de plazas públicas o los altos precios de estos recursos en el ámbito privado. Estos factores propician situaciones de informalidad ya sea en el cuidado familiar, o a través de personas cuidadoras informales. En este último caso esto es debido a que los cuidados dirigidos a personas mayores con movilidad reducida o muy mayores, de carácter profesional pueden tener precios muy altos, más aún cuando la atención es permanente y amplios sectores sociales no se los pueden permitir si no es con una ayuda complementaria del Estado (López-Lago et al., 2021a).

También el modelo "familista" de cuidados en España se ve perpetuado por la necesidad de solidaridad dentro del ámbito familiar a causa de que los desarrollos institucionales para la atención de los cuidados de larga duración no cubren las necesidades reales de una población cada vez más envejecida (Di Novi et al., 2015; Spijker \& Zueras, 2020). En este sentido varios autores coinciden en que la ejecución deficiente de la $\operatorname{LAPAD}^{2}$, como consecuencia de la crisis económica de 2008, tuvo como resultado la continuidad de la informalidad laboral de las personas cuidadoras, especialmente en las mujeres (De La Fuente et al., 2016; Moreno-Colom et al., 2017; Recio et al., 2015). Esta Ley aportaba mejoras sustanciales a la situación de las personas que se dedicaban a los cuidados de larga duración de las personas mayores asumiendo de forma decidida la responsabilidad del Estado en este tipo de cuidados (Moreno-Colom et al., 2017) lo que debería haber llevado consigo una amplia regulación profesional en el sector. Sin embargo, los severos problemas de financiación que

${ }^{2}$ LAPAD: Ley 39/2006, de 14 de diciembre, de Promoción de la Autonomía Personal y Atención a las personas en situación de dependencia, también llamada Ley de Dependencia. 
conllevó la crisis económica de 2008 imposibilitaron la ejecución adecuada de las prestaciones y servicios contemplados en la Ley (Spijker \& Zueras, 2020), dejando un sector muy inestable y con una alta precarización de sus trabajadores (Deusdad, 2020).

Este desarrollo institucional precario encaja en los modelos de Estado del Bienestar del Sur de Europa. Moreno-Colom et al. (2017) mencionan para el caso español, que los servicios que atienden a los cuidados de las personas mayores aprovechan la "cultura familiar del país" para su diseño. Estos autores señalan el carácter "híbrido" del modelo de atención a la dependencia español, con propuestas políticas que entroncan con la tradición socialdemócrata de los Estados del Bienestar, al tiempo que sus desarrollos han sido objeto de políticas liberales en las que la familia y la cultura son lo fundamental en los cuidados. Por el contrario, García-Faroldi (2015) ubica a España plenamente en el "familismo", propio de los modelos conservadores y mediterráneos, con un alto grado de informalidad en contraste con los modelos liberales y socialdemócratas sustentados en cuidados profesionales. En ambas posturas el carácter "familista" de la cultura de cuidados hacia las personas mayores propicia la informalidad laboral.

Además, Spijker y Zueras (2020) señalan como un factor de perduración de la informalidad en los cuidados, los altos índices de desempleo de las sociedades mediterráneas lo que por una parte aminora la capacidad de las familias para la contratación formal, al tiempo que propicia que estos trabajos se realicen dentro de la economía sumergida.

Otros factores de persistencia de la informalidad tienen que ver con las características de quien demanda los servicios, por ejemplo, Cantarero et al. (2019) destacan que vivir en una zona rural, ser mujer, y tener bajos ingresos, se asocia con la demanda de cuidados informales. Así mismo Spijker y Zueras (2020) consideran que la edad es un factor que influye en los cuidados, siendo así que entre los 65 y 79 años hay una preponderancia de la informalidad vinculada a los cuidados de la pareja dentro del hogar. Estos cuidados, cuando hay personas dependientes, normalmente se realizan con ayuda de puntual de servicios de asistencia a domicilio, pero con horarios muy limitados, suponiendo un complemento a la ayuda informal. No es así para los cuidados para las personas de más de 80 años, donde la forma de cuidado que se ha vuelto preponderante ha sido el recurso residencial.

Consecuentemente la informalidad de los cuidados hacia las personas mayores en España se sustenta en dos elementos fundamentales: por un lado, la inercia cultural y, por otro la debilidad del sistema del bienestar. Esto propicia altos niveles de informalidad en los cuidados, que recaen en la mayoría de las ocasiones en el núcleo familiar. Ahí encontramos que son las mujeres las principales proveedoras de cuidados. Éstas pueden ser parejas, principalmente antes de los 80 años, hijas, incluso nueras. También hay un tipo de informalidad con cuidadoras de fuera del núcleo familiar prestado por mujeres, en muchas ocasiones de origen inmigrante (Casado-Mejía et al., 2012; Oliva et al., 2017). En el siguiente apartado trataremos de ver cómo estos colectivos de cuidadoras informales se relacionan con la tecnología.

\footnotetext{
${ }^{3}$ Modelo de estado del bienestar donde la administración pública se apoya en el rol de las familias como institución central de
} los cuidados (Muñoz, 2015). 


\section{LOS CUIDADORES INFORMALES Y SU RELACIÓN CON LAS TECNOLOGÍAS EN ESPAÑA}

Los dos grupos, mujeres mayores que cuidan en el ámbito familiar y cuidadoras informales de origen extranjero, que han sido identificados como principales actores en la cultura de los cuidados informales tienen una relación difícil con el acceso a las tecnologías. Según el Barómetro de la eigualdad en España (Martínez, 2020) existen desigualdades digitales entre hombres y mujeres, especialmente en el ámbito laboral y las habilidades digitales, donde se encuentran patrones de desventaja para las mujeres, especialmente entre las nacidas entre 1951 y 1970. En el caso de grupos de más edad no hay tanta diferencia entre sexos en el acceso y uso de las TIC, pero sí hay una disminución notable comparado con el resto grupos más jóvenes. En el mismo sentido se expresan González et. al (2015) señalando la baja penetración de las TIC (Tecnologías de la información y la comunicación) en la población mayor de 65 años. Esto afecta de forma concreta al grupo de cuidadoras de más de 65 años, que ejercen su labor de cuidados dentro del núcleo familiar. Al mismo tiempo cabe señalar que el dispositivo más utilizado por personas mayores de 65 años en España es el dispositivo móvil (González et al., 2015). Por ello parece que, si se quieren desarrollar acciones formativas sobre cuidados dirigidas a estas personas, el teléfono móvil puede ser la herramienta más adecuada por ser la más generalizada.

Así mismo, para el colectivo de cuidadoras informales, de fuera del núcleo familiar, el acceso a las tecnologías puede plantear diversas dificultades, muchas veces vinculadas a la precariedad económica y los obstáculos para conseguir dispositivos adecuados y una buena conexión a la red. Como principio del que partir, el alto número de mujeres migrantes que participan en los cuidados informales en España (Casado-Mejía et al., 2012; Oliva, 2017) nos da una pista del camino para investigar su relación con las tecnologías estableciendo una relación con el colectivo migrante en general, ya que no se han encontrado datos desagregados por sexo en este sentido. En España las personas inmigrantes utilizan cada vez más las nuevas tecnologías, siendo constatable el predominio del teléfono móvil (Viruela, 2007). Riezu et al. ( 2014) afirman que las personas inmigrantes muestran unos niveles de uso de las TIC similares a los de la población autóctona. Destacan que el uso de las tecnologías en el colectivo inmigrante aumenta su capital social, aunque con un riesgo, que sea un capital social vinculante y cerrado. Esto es, que se refuercen las relaciones con los países de origen, así como con compatriotas en la sociedad de acogida intensificando el aislamiento de las comunidades por país al crearse comunidades virtuales que satisfacen las necesidades de interacción. Sin embargo, tal y como indica Viruela (2007) esta creación de comunidad a través de la tecnología también contribuye a la creación de redes de solidaridad que pueden incidir de forma positiva en el desarrollo y la inclusión social de las personas inmigrantes, incluso en el fomento del asociacionismo. Así pues encontramos que en el colectivo de cuidadoras informales de origen inmigrante podemos encontrar circuitos de comunicación a través de las tecnologías que pueden ser usados para la difusión de material formativo para las labores de cuidados. 
También puede ser de interés el señalar que los usos que le dan las mujeres a las TIC están vinculados a actividades relativas a la salud, a diferencia de los hombres donde predominan usos relativos a banca electrónica o comercio online (Martínez, 2020). Este uso diferencial puede facilitar la difusión de contenidos sobre cuidados hacia personas mayores en un sector tan feminizado como es el de los cuidados informales.

Desde el análisis de la literatura científica encontramos algunos puntos de partida que pueden ser útiles para que las administraciones públicas desarrollen estrategias dirigidas a transmitir contenidos formativos en materia de cuidados a los distintos colectivos de cuidadores informales. Sin duda la herramienta tecnológica más adecuada es el teléfono móvil por su amplia difusión entre los colectivos implicados. Así mismo las aplicaciones de mensajería instantánea pueden ser el medio más apropiado de difusión de esos contenidos dada su enorme expansión entre los usuarios de teléfonos móviles, siendo WhatsApp la preferida en España (Samaniego, 2019). Marilia Duque (2020) afirma que WhatsApp es una aplicación particularmente fácil de usar, con un diseño sencillo lo que le da un carácter inclusivo hacia personas poco familiarizadas con las tecnologías, como puede ser un amplio sector de las personas mayores cuidadoras. Asimismo, Duque (2020) destaca la existencia de una mayor probabilidad para atender los consejos de salud procedentes de una aplicación conocida y utilizada habitualmente por los usuarios que, de aplicaciones nuevas, las cuales tengan que ser instaladas y de las que desconozcan su funcionamiento. Estas ventajas del WhatsApp como difusor de información las ha utilizado la Organización Mundial de la Salud que ha desarrollado un servicio de alertas en WhatsApp sobre COVID-19 con una capacidad potencial para hacer llegar a 2000 millones de personas información veraz sobre la pandemia (WHO, 2020). Un buen ejemplo a tener en cuenta para las administraciones públicas españolas para difundir información sobre cuidados

Para el colectivo de los cuidadores informales de origen extranjero este servicio de mensajería ofrece dos ventajas evidentes. La primera, la disponibilidad en más de 60 idiomas en Android y 40 en iPhone (WhatsApp, n.d.). La segunda es la posibilidad de organizar grupos, y grupos de difusión por parte de los usuarios, lo que es muy funcional a la organización en grupos por nacionalidades, característica de las redes de socialización de los inmigrantes y facilita la difusión de mensajes (López-Lago et al., 2021a).

\section{CONCLUSIONES}

Del análisis de cómo se relacionan con las tecnologías de la comunicación los principales colectivos de cuidadores informales de personas de edad avanzada, mujeres mayores dentro del entorno familiar y mujeres migrantes, concluimos que las herramientas óptimas para hacerles llegar materiales formativos sobre cuidados son las aplicaciones de mensajería instantánea. De forma particular el WhatsApp, dado que está muy extendido entre estos grupos y su uso les resulta familiar. Los contenidos para difundir pueden tener el formato de mensajes, documentos e infografías, pero lo más didáctico serían videos de corta duración donde se aborden temas de cuidados 
pedagógicamente adaptados a personal sin una formación profesional. Se pueden tratar temas de nutrición, comunicación, técnicas de movilización manual, bienestar o adherencia terapéutica entre otros muchos. También se pueden abrir sistemas de alarmas para desmentir rumores o fake news que pueden afectar a los cuidados de las personas mayores, como ocurre en la actual pandemia de COVID-19.

Finalmente, para poder desarrollar estos materiales y la estrategia formativa, se hace necesaria una investigación etnográfica que aporte con más detalle el universo de relaciones existentes entre los cuidadores informales y su vínculo con las tecnologías, además de las necesidades específicas en cuanto a formación en materia de cuidados, para posteriormente elaborar un plan de intervención que sirva de guía a las administraciones públicas a la hora de utilizar las TIC para mejorar la formación de este colectivo.

\section{AGRADECIMIENTOS}

Este trabajo forma parte de los resultados del proyecto 4IE+ (0499-4IE PLUS 4 E) financiado por el programa Interreg V-A España-Portugal (POCTEP) 2014-2020.

\section{REFERENCIAS}

Abellan, A., Perez, J., Pujol, R., Sundstrom, G., Jegermalm, M., \& Malmberg, B. (2017). Partner care, gender equality, and Ageing in Spain and Sweden. International Journal of Ageing and Later Life, 11(1), 69-89. https://doi.org/10.3384/ijal.1652-8670.16-305

Cantarero, D., Pascual, M. \& Rodríguez, B. (2019). Differences in the provision of formal and informal care services after the implementation of the dependency act: the Spanish case. Papeles de trabajo del Instituto de Estudios Fiscales, 5, 1-36. https://dialnet.unirioja.es/servlet/articulo?codigo=7206075

Casado-Mejía, R., Ruiz-Arias, E., y Solano-Parés, A. (2012). El cuidado familiar prestado por mujeres inmigrantes y su repercusión en la calidad del cuidado y en la salud. Gaceta Sanitaria, 26(6), 547-553. https://doi.org/10.1016/j.gaceta.2012.01.012

Casanova, G., Lamura, G., \& Principi, A. (2017). Valuing and Integrating Informal Care as a Core Component of Long-Term Care for Older People: A Comparison of Recent Developments in Italy and Spain. Journal of Aging and Social Policy, 29(3), 201-217. https://doi.org/10.1080/08959420.2016.1236640

De La Fuente, Y.M., Sotomayor, E.M. y Martín, M.C. (2016). Vulnerabilidad sobrevenida en personas en situación de dependencia en España. Scripta Nova. Revista electrónica de Geografía y Ciencias Sociales, $X X(535), 1-29$.

De Souza, M.C. (2019). The imperative of caring for the dependent elderly person. Ciencia e Saude Coletiva, 24(1), 247-252. https://doi.org/10.1590/1413-81232018241.29912018

Deusdad, B. (2020). COVID-19 and Nursing Homes ' crisis in Spain : Ageism and Scarcity of Resources . Research on Ageing and Social Policy, 8(2), 142-168. https://doi.org/10.447/rasp.2020.5598 
Di Novi, C., Jacobs, R., \& Migheli, M. (2015). The Quality of Life of Female Informal Caregivers: From Scandinavia to the Mediterranean Sea. European Journal of Population, 31(3), 309-333. https://doi.org/10.1007/s10680-014-9336-7

Duque, M. (2020). Learning from WhatsApp Best Practices for Health Learning from WhatsApp Best Practices for Health. Communication protocols for hospitals and medical clinics. ASSA.

Durán, M. (2015). La regulación de la dependencia en el derecho interno de la Unión Europea. Modelos de protección ciudadana para los residentes en la Unión Europea. Revista de Derecho Migratorio y Extranjería, 38, 317-337. https://dialnet.unirioja.es/servlet/articulo?codigo=5104895

Fernández-Alonso, M. \& Ortega, M. (2018). Gender and informal social support in Spanish culture. Research on Ageing and Social Policy, 6(2), 118-146. https://doi.org/10.17583/rasp.2018.3212

García-Faroldi, L. (2015). Welfare States and Social Support: An International Comparison. Social Indicators Research, 121(3), 697-722. https://doi.org/10.1007/s11205-014-0671-1

González, C., Fanjul, C. y Cabezuelo, F. (2015). Uso, consumo y conocimiento de las nuevas tecnologías en personas mayores en Francia, Reino Unido y España. Comunicar, XXIII(45), 1928. https://doi.org/10.3916/C45-2015-02

Instituto de Mayores y Servicios Sociales [MSERSO] (2005). Cuidados a las Personas Mayores en los Hogares Españoles. El entorno familiar. Ministerio de Trabajo y Asuntos Sociales. http://www.imserso.es/InterPresent1/groups/imserso/documents/binario/cuidadosppmmhogares. pdf

López, E. (2016). Puesta al día: cuidador informal. Revista de Enfermeria CyL, 8(1), 71-77. http://www.revistaenfermeriacyl.com/index.php/revistaenfermeriacyl/article/view/164

López-Lago, L., Arroyo, S., Cipriano, C., Bonilla, J. \& Muñoz, B. (2021a). Technological Solutions and Informal Care Culture for the Elderly: An Intervention Proposal for Training Actions. In C. GarciaAlonso, Jose; Fonseca (Ed.), Lecture Notes in Bioengineering (Vol. 1, pp. 315-323). Springer International Publishing. https://doi.org/10.1007/978-3-030-72567-9_29

López-Lago, L., Arroyo, S., Cipriano, C., Luengo, J. \& Muñoz, B. (2021b). Technology in the Face of the Challenges of the Long-Term Care System for the Elderly in Spain. In C. García-Alonso, José; Fonseca (Ed.), Lecture Notes in Bioengineering (Vol. 1). Springer International Publishing. https://doi.org/10.1007/978-3-030-72567-9_34

Martínez, J.L. (coord). (2020). Nuestras vidas digitales. Barómetro de e-igualdad de género en España. Ministerio de Igualdad. Ministerio de Asuntos Económicos y Transformación Digital.https://www.mineco.gob.es/stfls/mineco/ministerio/igualdad/ficheros/NuestrasVidasDigital esEdicAbril2020.pdf

Martinez, J.R. (2003). Cuidados informales en España. Problema de desigualdad. Revista de Administración Sanitaria Siglo XXI, 1(2), 275-288.

Moreno-Colom, S., Recio, C., Torns, T., \& Borràs, V. (2017). Long-term care in Spain: Difficulties in professionalizing services. Journal of Women and Aging, 29(3), 200-215. https://doi.org/10.1080/08952841.2015.1125699

Moreno, A. (2004). El familiarismo cultural en los Estados del Bienestar del Sur de Europa: transformaciones de las relaciones entre lo público y lo privado. Revista Sistema, 182, 200-215. 
Muñoz, O. (2015) El declive de la Ley de Dependencia. Familismo implícito y oportunidad perdida en la profesionalización de los cuidados. Encrucijadas, 10, n1001, 1-16. https://dialnet.unirioja.es/servlet/articulo?codigo $=5418697$

Oliva, P.L., Ordóñez, L. y Peinado, P. (2017). Análisis de género desde la enfermería en cuidadoras inmigrantes de personas mayores. ENE. Revista de Enfermería, 11(1). http://eneenfermeria.org/ojs/index.php/ENE/article/view/646/cuidadores

Osorio, L., Salinas, F., \& Cajigas, M. (2018). Responsabilidad social y bienestar de la persona mayor. CIRIEC-España, Revista de Economía Pública, Social y Cooperativa, 92, 223-252. https://doi.org/10.7203/ciriec-e.92.8959

Recio, C., Moreno-Colom, S., Borràs, V. \& Torns, T. (2015). La profesionalización del sector de los cuidados. Zerbitzuan, 60, 179-194. https://doi.org/10.5569/1134-7147.60.12

Riezu, X., Oiarzabal, P., Aretxebala, M.E. y Maiztegui, C. (2014). El uso de las TIC por parte de los migrantes y sus consecuencias para el capital social. In Crisis y cambio. Propuestas desde la Sociología: actas del XI Congreso Español de Sociología. Universidad Complutense de Madrid. (pp. 1370-1378).

Samaniego, R. (2019). Panel Hogares CNMC: Los españoles llaman desde el móvil y (queman) el Whatsapp. Blog de La Comisión Nacional de Los Mercados y La Competencia. https://blog.cnmc.es/2019/10/31/los-espanoles-llaman-desde-el-movil-y-queman-el-whatsapppanel-hogares-cnmc/

Spijker, J., \& Zueras, P. (2020). Old-Age Care Provision in Spain in the Context of a New System of Long-Term Care and a Lingering Economic Crisis. Journal of Population Ageing, 13(1), 41-62. https://doi.org/10.1007/s12062-018-9232-8

Viruela, R. (2007). Migración y nuevas tecnologías de la información y la comunicación: inmigrantes rumanos en España. Migraciones, 21(2007), 259-290.

WhatsApp. (n.d.). Cómo cambiar el idioma de WhatsApp. Retrieved September 13, 2020, from https://faq.whatsapp.com/general/account-and-profile/how-to-change-whatsappslanguage/?lang=es

World Health Organization [WHO]. (2020). WHO Health Alert brings COVID-19 facts to billions via WhatsApp (2020). World Health Organization Newsroom 20/03/2020. https://www.who.int/newsroom/feature-stories/detail/who-health-alert-brings-covid-19-facts-to-billions-via-whatsapp

Zabalegui, A., Juandó, C., Sáenz de Ormijana, A., Ramírez, A.M., Pulpón, A., López, L., Bover, A., Cabrera, E., Corrales, E., Gallart, A., González M.A., Gual, M.P., Izquierdo, M.D. y Díaz M. (2007). Los ciudadores informales en España: perfil y cuidados prestados. Rol de Enfermería, 30(7-8), 33-38. 